\title{
Ultra-sonografia Transvaginal com Doppler Colorido para Seleção das Pacientes para Tratamento Conservador da Gravidez Ectópica Íntegra
}

Transvaginal Ultrasonography with Color Doppler to Select the Patients for Conservative Treatment of Unruptured Ectopic Pregnancy

Julio Elito Junior, André Reichmann, Mary N. Uchiyama, Luiz Camano

\section{RESUMO}

Objetivo: avaliar a eficiência da ultra-sonografia (US) transvaginal com Doppler colorido para a previsão dos resultados do tratamento sistêmico da gravidez ectópica integra com dose única de metotrexato.

Pacientes e método: vinte pacientes com diagnóstico de gravidez ectópica foram incluidas no estudo. Os critérios de inclusão foram: estabilidade hemodinâmica, massa anexial com diâmetro $\leq 5,0 \mathrm{~cm}$ e queda dos titulos de beta-hCG inferior a 15\% num intervalo de 24 horas. Foram excluidas as pacientes que apresentaram doença hepática ou renal ou discrasias sangüineas. O acompanhamento foi realizado com dosagens seriadas de beta-hCG nos dias 4 e 7 após instituido o tratamento, e semanalmente até que os titulos de beta-hCG se tornassem negativos. As pacientes foram classificadas em três conjuntos, conforme a US com doppler colorido: elevado risco (fluxo trofoblástico acometendo mais de 2/3 da massa anexial), médio risco (quando o fluxo trofoblástico comprometia de 1/3 a 2/3 do anel tubário) e de baixo risco (fluxos que acometiam menos de 1/3 da massa anexial).

Resultados: o sucesso do tratamento com dose única de MTX foi de 75\% (15 pacientes); quando empregamos uma segunda dose, a percentagem de bons resultados foi de $85 \%$. Confrontamos os resultados obtidos pela US transvaginal e Doppler colorido com o sucesso e o insucesso da terapêtica. Observamos que, das 20 pacientes, 4 apresentaram Doppler de elevado risco e todas evoluíram com fracasso, ao passo que em 16 casos o Doppler foi classificado como de médio ou baixo risco, sendo que destes 15 evoluiram com sucesso.

Conclusão: a ultra-sonografia transvaginal com Doppler colorido classificado como de elevado risco indica situação desfavorável para o tratamento medicamentoso da gravidez ectópica com dose única de metotrexato, ao passo que o Doppler de médio e baixo risco indica situação favorável para o tratamento clínico, porém estes resultados devem sempre ser analisados em conjunto com a curva de evolução dos títulos de beta-hCG.

PALAVRAS-CHAVE: Ultra-sonografia. Dopplervelocimetria. Gravidez ectópica. Quimioterapia.

Disciplina de Obstetrícia da Universidade Federal de São Paulo - Escola Paulista de Medicina e Departamento de Diagnóstico por Imagem da Universidade Federal de São Paulo - EPM

Correspondência:

Julio Elito Junior

Rua Sampaio Vidal, 824

01443-000 - Jardim Paulistano - São Paulo

Fone: (011) 852-4769
Introdução

A gravidez ectópica (GE) tem sido alvo nos últimos anos de investigações que alteraram radicalmente a óptica com que era analisada pelos tocoginecologistas. Destacamos a importância na realização do diagnóstico precoce, o que propicia o 
emprego de condutas conservadoras. O diagnóstico atualmente é feito de forma precoce graças ao advento das dosagens quantitativas de beta-hCG (fração beta do hormônio gonadotrópico coriônico) e do emprego da ultra-sonografia (US) transvaginal. Lançando mão destes dois recursos e analisando os resultados em conjunto, em aproximadamente $100 \%$ dos casos chegaremos ao diagnóstico de gravidez ectópica íntegra ${ }^{1}$.

Outro método propedêutico que podemos empregar no manejo da GE é a ultra-sonografia transvaginal com Doppler colorido. Apesar de que na grande maioria dos casos o exame de US em escala de cinza seja suficiente, alguns autores empregando o Doppler colorido, obtiveram uma sensibilidade de $95 \%$ e uma especificidade de $98 \%$ no reconhecimento da $\mathrm{GE}^{8}$. O exame consiste inicialmente na análise do grau de vascularização uterina e de presença ou ausência de fluxo trofoblástico periendometrial na cavidade uterina com o intuito de se descartar uma eventual gravidez tópica viável ou não. Na ausência de fluxo nesta região, parte-se para análise dos ovários, observando a presença ou ausência de fluxo ao redor do corpo lúteo. Por fim é avaliada a vascularização na massa anexial, que pode apresentar-se com diversos aspectos, sendo o mais característico o fluxo trofoblástico que representa um fluxo arterial de baixa resistência, ou seja de resistência inferior a $0,45^{7}$.

A ultra-sonografia transvaginal com Doppler colorido tem o seu papel no diagnóstico da gravidez ectópica, mas a sua função mais importante é avaliar o grau de vascularização da massa anexial para orientar e acompanhar a evolução das pacientes submetidas ao tratamento sistêmico com dose única de metotrexato ${ }^{2,3,4}$.

O objetivo do nosso estudo é avaliar a importância da ultra-sonografia transvaginal com Doppler colorido na seleção dos casos para o tratamento sistêmico da gravidez ectópica com dose única de metotrexato (50 $\left.\mathrm{mg} / \mathrm{m}^{2} \mathrm{IM}\right)$.

\section{Pacientes e Método}

Foi realizado estudo prospectivo na Disciplina de Obstetrícia da Universidade Federal de São Paulo - Escola Paulista de Medicina com a inclusão de 20 pacientes com gravidez ectópica integra, no período de outubro de 1995 a dezembro de 1996.

O diagnóstico foi feito analisando os dados clínicos, associado à propedêutica subsidiária, como a dosagem sérica de beta-hCG, a US transvaginal e o Doppler colorido.

A ultra-sonografia foi realizada inicialmente pelo autor, sendo complementada posteriormente com as informações adicionais obtidas por médico radiologista da Disciplina de Diagnóstico por Imagem da UNIFESP-EPM. O equipamento utilizado foi o ATL UM-9 (Bothel, Wash) com transdutor de banda larga de $5-9 \mathrm{MHz}$ para via transvaginal. Após minucioso exame em escalas de cinza, utilizamos o Doppler colorido para o mapeamento vascular do útero, ovários e eventuais massas anexiais, seguindo-se por último a análise espectral dos fluxos encontrados nos anexos e, quando necessário, da região periendometrial.

Analisamos no exame, em escala de cinza, a cavidade uterina, estudando a espessura endometrial e sua ecogenicidade, a presença de saco gestacional e/ou restos placentários. Nos ovários, procuramos identificar sempre que possivel o corpo lúteo.

Na US com Doppler colorido seguimos uma sistematização: avaliamos primeiramente o grau de vascularização uterina e presença ou ausência de fluxo trofoblástico periendometrial, com o intuito de descartar gravidez tópica (viável ou inviável). Posteriormente observamos a presença ou ausência de corpo lúteo, bem como efetuamos a análise da resistência a estes fluxos. Após o estudo da vascularização uterina e do corpo lúteo, partimos para a análise do objetivo de nosso estudo, que é a vascularização da massa anexial. Esta é verificada segundo os critérios descritos por Pellerito et al. ${ }^{8}$.

A resistência do fluxo foi avaliada pelo índice de resistência (IR).

A natureza (arterial ou venosa) foi avaliada de acordo com a morfologia da onda.

Caracterizou-se como fluxo trofoblástico a presença de fluxo arterial de baixa resistência ao redor da massa, com índice de resistência menor que 0,45 . A morfologia da onda é típica, com queda lenta do fluxo diastólico e pico sistólico superior a $10 \mathrm{~cm} / \mathrm{s}^{7}$. Para confirmação desse fluxo como trofoblástico, avaliamos a região anexial, distinguindo-a do ovário, para não confundir com o fluxo do corpo lúteo.

Estudos dopplervelocimétricos foram classificados em três grupos de risco para insucesso do tratamento: elevado, médio e baixo risco. O de elevado risco caracteriza-se por fluxo trofoblástico em mais de $2 / 3$ da massa anexial ${ }^{3}$; o de médio risco, quando compromete de $1 / 3$ a $2 / 3$ do anel tubário (Figura 1); classificamos de baixo risco as seguintes situações: fluxos que acometem menos de 1/3 da massa anexial, ausência de 
vascularização ao redor da massa ou presença de fluxos arteriais de resistência aumentada (IR maior que 0,5$)^{4}$. A presença de fluxos de morfologia bizarra que se apresentam com importante alargamento espectral, com tempos de aceleração longos e diástoles ausentes ou negativas ${ }^{7,9}$, e o aumento da vascularização venosa ao redor da massa com formação de lagos venosos foram considerados como prováveis sinais de involução trofoblástica.

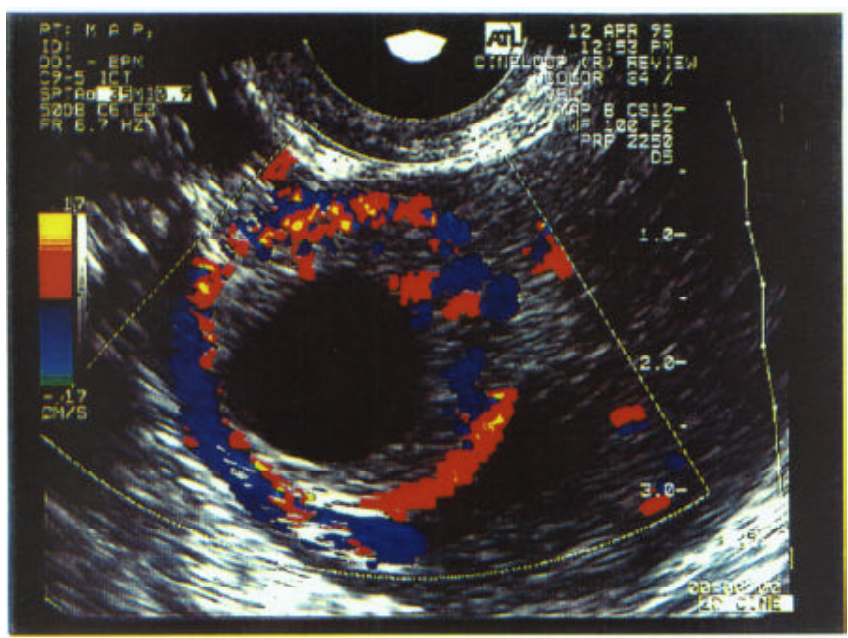

Figura 1 - Caso de gravidez tubária apresentando fluxo trofoblástico que acomete todo 0 anel tubário (doppler de elevado risco).

Além destes exames mencionados, solicitamos rotineiramente a tipagem sangüinea, fator $\mathrm{Rh}$, hemograma completo, transaminases $\mathrm{e}$ creatinina. A tipagem do grupo sangüineo e o fator Rh têm por finalidade preparar a equipe para eventual hemotransfusão. Impõe-se também, em relação às gestantes $\mathrm{Rh}$ negativo, avaliar a necessidade de ministrar imunoglobulina antiRh (na dose de 300 microgramas), quando o teste de Coombs indireto não revelar isoimunização prévia.

Firmado o diagnóstico de GE íntegra, verificamos alguns critérios antes de tomarmos a conduta. Os critérios de inclusão foram: a estabilidade hemodinâmica, o diâmetro da massa anexial igual ou menor que $5,0 \mathrm{~cm}$, o desejo de gravidez futura e a concordância em participar do estudo, mediante consentimento escrito, em documento formulado e aprovado pela Comissão de Ética Médica da UNIFESP-EPM.

Os critérios de exclusão foram: as pacientes necessitadas de hemotransfusão ou com reconhecida sensibilidade ao MTX e as evidências de doença hepática, renal ou supressão da medula óssea. Assim, não fizeram parte do protocolo aquelas com discrasias sangüineas, como leucopenia (número de glóbulos brancos menor que $2.000 \mathrm{cel} / \mathrm{mm}^{3}$ ) e trombocitopenia (número de plaquetas menor que $100.000 / \mathrm{mm}^{3}$ ). Também não participaram do protocolo as que apresentaram aminotransferase da alanina e do aspartato alterada ou doença renal (creatinina maior que $1,5 \mathrm{mg} / \mathrm{dl}$ ); quando houve queda dos títulos de betahCG superior a 15\% num intervalo de 24 horas, estas pacientes fizeram parte do protocolo de outro estudo, o da conduta expectante.

As pacientes foram tratadas inicialmente com ministração de dose única de MTX (50 mg/ $\mathrm{m}^{2}$ de superficie corporal por via intramuscular) e, quando indicado, complementação com nova dose. Na avaliação clínica, investigamos o aparecimento dos efeitos colaterais da medicação. As pacientes também foram cuidadosamente examinadas para detectarmos precocemente sinais e sintomas de complicação da GE.

O acompanhamento se fez por meio de dosagens de beta-hCG, realizadas no momento da ministração do MTX, no quarto e no sétimo dia após o emprego da droga. As que apresentaram queda dos títulos de beta-hCG maiores que $15 \%$, apurados no $4^{\circ}$ e no $7^{\circ}$ dia, foram classificadas como sucesso do tratamento e foram seguidas com dosagens semanais de beta-hCG, até a queda dos valores a niveis pré-gravídicos. Quando a queda foi inferior a $15 \%$, avaliadas no $4^{\circ}$ e no $7^{\circ}$ dia, consideramos como insucesso do tratamento e ministramos, no sétimo dia, nova dose de MTX, seguindo a mesma sistematização ${ }^{6,11}$

As relações sexuais foram permitidas após o retorno dos valores de beta-hCG a niveis inferiores a $5 \mathrm{mUI} / \mathrm{ml}$, orientando-se então para o uso de anticoncepcional oral ou do método de barreira, por um periodo de 3 meses aproximadamente, pois neste prazo, geralmente, ocorre o desaparecimento da imagem ultrasonográfica da massa anexial ${ }^{12}$.

Como controle, foi realizada a histerossalpingografia por volta do $3^{\circ}$ mês após o fim do tratamento. Posteriormente, as pacientes foram acompanhadas semestralmente, para observação da ocorrência de nova gravidez.

Para a análise do resultado foi utilizado teste não-paramétrico, levando-se em consideração a natureza das variáveis estudadas. Foi aplicado o teste exato de Fisher para tabelas $2 \times 2$ (Siegel) ${ }^{10}$, com o objetivo de estudar as associações entre Doppler colorido de alto risco e o sucesso ou insucesso do tratamento.

Neste teste fixou-se em 0,05 , ou $5 \%$, o nivel de rejeição da hipótese de nulidade, assinalandose nos resultados os valores significantes. 


\section{Resultados}

Das 20 pacientes submetidas ao tratamento medicamentoso com MTX, 75,0\% (15/20) evoluíram com êxito em face da primeira dose de MTX; a associação de uma segunda dose aumentou o índice de bons resultados para $85,0 \%$ $(17 / 20)$.

Das 20 pacientes tratadas com dose única de MTX, observou-se Doppler de elevado risco em 4 e todas evoluíram com insucesso. O Doppler de médio e baixo risco foi observado em 16 casos sendo que, com exceção de 1 paciente, todas evoluíram com sucesso (Tabela 1). O teste exato de Fisher mostrou associação significante entre o Doppler de elevado risco e o insucesso do tratamento. Portanto, a presença de exuberante fluxo trofoblástico, ou seja, o Doppler de elevado risco, é uma situação de agravo para o tratamento medicamentoso.

Tabela 1 - Correlação entre o sucesso ou insucesso do tratamento de GE íntegra com dose única de metotrexato $\left(50 \mathrm{mg} / \mathrm{m}^{2} \mathrm{IM}\right)$ e os dados da US transvaginal com Doppler colorido.

\begin{tabular}{lcccc}
\hline Doppler Colorido & \multicolumn{2}{c}{ Tratamento } & Total & $\%$ Sucesso \\
& Sucesso* & Insucesso & & \\
\hline Elevado Risco & 0 & 4 & 4 & 0,0 \\
Baixo + Médio Risco & 15 & 1 & 16 & 93,8 \\
Total & 15 & 5 & 20 & 75,0 \\
\hline
\end{tabular}

Teste exato de Fisher

$p=0,0010^{*}$ ou $0,10 \%$

\section{Discussão}

Classicamente o diagnóstico definitivo e o tratamento da gravidez ectópica eram realizados durante a laparotomia ou a laparoscopia. Com a possibilidade de se realizar o tratamento medicamentoso da GE em casos selecionados, podemos evitar o emprego de métodos invasivos para o diagnóstico. Além disso, quando instituímos o tratamento clínico necessitamos lançar mão de exames subsidiários que possam nos ajudar a avaliar se o caso em questão evoluirá bem ou mal com a conduta tomada. A ultra-sonografia transvaginal com Doppler colorido é um bom parâmetro para se avaliar a gravidade do caso e estabelecer um prognóstico em relação ao sucesso do tratamento.

Antes de ser instituído o tratamento conservador é importante diferenciar uma gravidez ectópica em evolução de outra em regressão. Pacientes com GE em evolução apresentam títulos elevados de beta-hCG, além de demonstrarem, ao exame de Doppler colorido, o índice de resistência e pulsatilidade mais baixos. Nos casos com GE em involução os títulos de beta-hCG são geralmente mais baixos e o indice de resistência e pulsatilidade da massa anexial são mais elevados. Desta forma, Bonilla-Mussoles et al. ${ }^{4}$ demonstraram que o mapeamento vascular da massa anexial avaliado pelo Doppler colorido pode diferenciar uma gravidez ectópica em evolução de outra em involução.

Acreditamos que o exame de Doppler espelha a evolução dos títulos de beta-hCG, ou seja, títulos elevados e em ascensão estão associados a Doppler de elevado risco e, portanto, mais susceptiveis ao fracasso do tratamento medicamentoso, o que foi comprovado em nosso estudo, já que 4 casos classificados pelo Doppler como de elevado risco evoluíram com insucesso. Em nosso trabalho, a maioria dos casos $(15 / 16)$ com niveis de beta-hCG baixos ou em regressão e relacionados com o Doppler de médio e baixo risco, evoluíram com sucesso após tratamento sistêmico com dose única de MTX.

As dosagens séricas de beta-hCG são demoradas, ao passo que a análise do fluxo vascular da massa anexial com Doppler colorido apresenta resultado imediato. Desta forma, o emprego da ultra-sonografia transvaginal com Doppler colorido ao invés das dosagens de beta-hCG pode dar de forma mais rápida o diagnóstico de $\mathrm{GE}$ e, como conseqüência, podemos instituir de forma mais precoce o tratamento medicamentoso com MTX, diminuindo o risco de ruptura tubária ${ }^{7}$.

Em nosso estudo, aguardamos em todos os casos os resultados das dosagens séricas de betahCG, mas realizamos de forma paralela a US transvaginal com Doppler colorido com o intuito de antecipar a gravidade do caso. A US transvaginal com Doppler colorido foi um parâmetro importante para orientar o tratamento clínico. Ressaltamos que o seu grande valor se concentra em aguardarmos 24 horas para a realização da segunda dosagem de beta-hCG ${ }^{5}$. Se o Doppler indicar médio ou baixo risco, teremos tranqüilidade de aguardar a segunda dosagem de beta-hCG em 24 horas, e a chance de sucesso do tratamento clínico é muito grande. Por outro lado, se o Doppler indica risco elevado, a vigilância sobre a paciente tem que ser redobrada até que se completem 24 horas para uma nova dosagem de beta-hCG, além do que a probabilidade de sucesso do tratamento é bem menor. Se tivéssemos um maior número de casos com Doppler de elevado risco, em nosso estudo, que evoluíssem com fracasso, poderiamos indicar enfaticamente que estas situações seriam contra-indicações para o 
tratamento clínico.

A US transvaginal com Doppler colorido é, portanto, um parâmetro importante na orientação do tratamento não-cirúrgico da gravidez ectópica, mas a sua avaliação em associação com a curva de evolução do beta-hCG é fundamental para o bom manejo do tratamento clínico das pacientes com esta condição.

\section{SUMMARY}

Purpose: to evaluate the efficacy of color Doppler in the prediction of results of the systemic treatment of unruptured ectopic pregnancy with a single dose of methotrexate.

Methodology: twenty patients with a diagnosis of ectopic pregnancy were included in the study. The inclusion criteria were: hemodynamic stability, adnexal mass $\leq 5.0 \mathrm{~cm}$ and decline of the titers of beta-hCG less than $15 \%$ in an interval of $24 \mathrm{~h}$. The exclusion criteria were hepatic or renal disease and blood dyscrasias. Follow-up was by serial determinations of beta-hCG on days 4 and 7 after the beginning of the treatment, and weekly until the titers were negative. The patients were classified into 3 groups according to color Doppler: high risk (trophoblastic flow covering more than $2 / 3$ of the mass), medium risk (when trophoblastic flow compromised $1 / 3$ to $2 / 3$ of tubal mass) and low risk (when trophoblastic flow covered less than $1 / 3$ of the mass).

Results: the success of the treatment with a single dose was $75 \%(15 / 20) ;$ when a second dose of MTX was used, the success rate was $85 \%$. When comparing color Doppler with the results of the medical treatment, we had high risk in 4 patients and in all the treatment failed; medium and low risk in 16 patients, and in 15 the treatment was successful.

Conclusion: color Doppler showing high risk indicated an unfavorable situation for the medical treatment with MTX, while medium and low risk in color doppler were favorable situations for the clinical treatment. However, these results should always be analyzed in association with the evolution curve of the beta-hCG titers.

KEY WORDS: Ultrasound. Dopplervelocimetry. Ectopic pregnancy.

\section{Referências Bibliográficas}

1. Ankum WM, Van Der Veen F, Hamerlynck JVT, Lammes FB. Transvaginal sonography and human chorionic gonadotrophin measurements in suspected ectopic pregnancy; a detailed analysis of a diagnostic approach. Hum Reprod 1993; 8:1307-11.

2. Atri M, Bret PM, Tulandi T, Senterman, MK. Ectopic pregnancy: evolution after treatment with transvaginal methotrexate. Radiology 1992; 185: 749-53.

3. Atri M, Bret PM, Tulandi T. Spontaneous resolution of ectopic pregnancy: initial appearance and evolution at transvaginal US. Radiology 1993; 186: 83-6.

4. Bonilla-Mussoles FM, Ballester MJ, Tarin JJ, Raga F, Osborne NG, Pellicer A. Does transvaginal color doppler sonography differentiate between developing and involuting ectopic pregnancies? J Ultrasound Med 1995; 14:175-81.

5. Brown DL, Felker RE, Stovall TG, Emerson DS, Ling FW. Serial endovaginal sonography of ectopic pregnancies treated with methotrexate. Obstet Gynecol 1991; 77: 406-9.

6. Elito Junior J, Uchiyama M, Camano L. O metotrexate no tratamento sistêmico da prenhez ectópica íntegra. Rev Bras Ginecol Obstet 1996; 18: 537-41.

7. Emerson DS, Cartier MS, Altieri LA, Felker RE, Smith WC, Stovall TG, Gray LA. Diagnostic efficacy of endovaginal color Doppler flow imaging in an ectopic pregnancy screening program. Radiology 1992; 183: 413-20.

8. Pellerito JS, Taylor KJW, Quedens Case C, Hammers LW, Scoutt LM, Ramos IM, Meyer WR. Ectopic pregnancy: evaluation with endovaginal color flow imaging. Radiology 1992; 183: 407-11.

9. Reichmann AP, Elito Junior J, Lederman HM. Ectopic pregnancy: Further experience with the so-called "Bizarre waveforms". J Ultrasound Med 1997; 16: S79.

10.Siegel S. Estatística no parametrica. Ed. Trillas México, Ed. Trielas; 1875. p. 346.

11.Stovall TG, Ling FW. Single dose of methotrexate: an expanded clinical trial. Am J Obstet Gynecol 1993; 168: 1759-65.

12.Stovall TG, Ling FW, Gray LA. Single dose methotrexate for treatment of ectopic pregnancy. Obstet Gynecol 1991; 77: 754-7. 